\title{
Design of Faraday cup ion detectors built by thin film deposition
}

2

3 G.A. Szalkowski ${ }^{\mathrm{a}}$, D.S. Darrow ${ }^{\mathrm{b}}$, F.E. Cecil ${ }^{\mathrm{c}}$

4 30332,gszalkowski3@gatech.edu discrete foil stack implementation.

\section{Introduction}

${ }^{a}$ Department of Nuclear Engineering, Georgia Institute of Technology, 770 State St., Atlanta, GA

${ }^{\mathrm{b}}$ Princeton Plasma Physics Laboratory, P. O. Box 451, Princeton, NJ, 08543, ddarrow@pppl.gov

${ }^{\mathrm{c}}$ Department of Physics, Colorado School of Mines, Golden, CO 80401, fcecil@mines.edu

Abstract: Thin film Faraday cup detectors can provide measurements of fast ion loss from magnetically confined fusion plasmas. These multilayer detectors can resolve the energy distribution of the lost ions in addition to giving the total loss rate. Prior detectors were assembled from discrete foils and insulating sheets. Outlined here is a design methodology for creating detectors using thin film deposition that are suited to particular scientific goals. The intention is to use detectors created by this method on the Joint European Torus (JET) and the National Spherical Torus Experiment-Upgrade (NSTX-U). The detectors will consist of alternating layers of aluminum and silicon dioxide, with layer thicknesses chosen to isolate energies of interest. Thin film deposition offers the advantage of relatively simple and more mechanically robust construction compared to other methods, as well as allowing precise control of film thickness. Furthermore, this depositional fabrication technique places the layers in intimate thermal contact, providing for three-dimensional conduction and dissipation of the ionproduced heating in the layers, rather than the essentially two-dimensional heat conduction in the

For magnetically confined fusion plasmas, good confinement of energetic ions, whether neutral beam ions, ion cyclotron heated tail ions, or alpha particles produced by deuterium-tritium (DT) fusion, is important for efficient heating, as well as to protect the plasma-facing walls from unnecessary damage. Energies of these sorts of ions typically range from a few tens of keV for neutral beam ions to several MeV for DD and DT fusion products and also for ion cyclotron heated energetic tail ions. Measuring the ions that do escape the plasma can give vital insights as to which conditions promote good ion confinement, and can provide some information regarding 
which internal plasma processes can lead to ion loss. Faraday cup detectors have been used for this purpose on several occasions ${ }^{1-5}$, including thin foil detectors in the Joint European Torus (JET) ${ }^{6}{ }^{6}$ The principle of operation is simple: energetic ions which are on lost trajectories will leave the plasma volume and strike the wall of the confinement vessel. This then results in a net current to the wall. If the loss location or locations are suitably equipped with an ion collector plate (the Faraday cup) and appropriate electronic detection, the loss rate(s) as a function of time can be measured. Furthermore, a stack of multiple, electrically insulated conductive layers at the same position can provide some crude energy resolution of the lost ions as lower energy ions will stop near the surface, while more energetic ions deposit their current in deeper layers. The first and second generation JET fast ion loss detector designs involved assembling the layered detector from discrete foils and insulating sheets. The JET instrument design proposed below is intended to replace some of these currently existing thin foil detectors, and will work in conjunction with a previously installed scintillator detector ${ }^{7}$. The energy response of a deposited Faraday cup will be identical to that of one built by stacked thin layers, provided the corresponding layers in each detector are of the same composition and thickness. Construction of detectors using thin film deposition has also been proposed in prior work ${ }^{8,9}$. Using thin film deposition would allow for the construction of a detector as a single piece, much reducing the contact heat resistance between layers, which, in turn, would allow for three dimensional conduction and dissipation of ion induced heating. The resulting detector would also be more mechanically sound than those using the older design. (Indeed, it is believed that some of the existing JET detectors have failed due to cracking or breakage of the mica insulating sheets within, allowing adjoining foils to come into electrical contact.) Thermal management and mechanical durability are important issues as techniques for fast ion loss measurement in the International Thermonuclear Experimental Reactor (ITER) ${ }^{10}$ are evaluated. The use of thin film deposition also gives a considerably larger amount latitude of control as to the thicknesses of the foils, providing much better discrimination between specific energy ions, as will be discussed below. An important point of this present work is, we believe, that this technique of detector construction affords multiple operational benefits for the detector, including better energy resolution, in addition to those already cited. In addition, detectors for a wide range of experimental conditions for magnetically confined fusion plasmas can be fabricated using a very modest set of materials, which are familiar in a range of industrial and scientific settings.

In the National Spherical Torus Experiment-Upgrade (NSTX-U) ${ }^{11}$, the fast ions of interest are the neutral beam ions, typically $90 \mathrm{keV}$ deuterons. While thermal stress on detectors is expected to be less severe than that in JET or ITER, the lower energy of the fast ions requires the layers in a Faraday cup detector to be so thin (hundreds of nm typical thickness) that stacking of free standing foils becomes mechanically impossible. Hence, detectors formed by thin film deposition are also desirable for application in NSTX-U or any other experiment with fast ion energies below $\sim 500 \mathrm{keV}$. 
2 Design Goals

Two variations of thin film Faraday cup detectors have been designed and are reported here; one for the measurement of neutral beam ions on NSTX-U and the other for the measurement of alpha particles produced by DT fusion reactions in JET. The design goal common to both detectors is to provide some level of energy resolution for lost particles. In the case of NSTX-U, with $90 \mathrm{keV}$ deuteron beam ions, this translates into a desire to discriminate between the onethird, one-half, and full energy ions with less than $25 \%$ uncertainty. The JET detector goal is to have enough resolution to show the energy distribution of the lost alpha particles with $\sim 30$ percent accuracy. Both designs would also have to operate at elevated vessel temperatures $\left(150^{\circ}\right.$ $\mathrm{C}$ for NSTX-U and $250^{\circ} \mathrm{C}$ for JET), and in an intense neutron/gamma radiation environment.

85 Thin foil Faraday cups have previously shown to have high immunity to neutron and gamma radiation. $^{12}$ The designs also had to include a sufficient thickness of insulation between the foils to prevent a large incidence of defects that would cause shorts between foils. This is counterbalanced by the need for the insulators to be thin enough so that the resolution of the detector would not be greatly diminished by ions stopping in the insulators instead of the conducting layers.

\section{Design Methodology}

Initial designs for both detectors were formulated from prior detectors, in the case of JET, or from simple layer thickness estimates based upon the known stopping powers of several metals for the ion species and energies of interest (NSTX-U). These initial designs were then evaluated and refined using the Stopping and Ranges of Ions in Matter code ${ }^{13}$ (SRIM) to optimize the material choices and thicknesses for each layer. SRIM was utilized because it has been exhaustively benchmarked over the last four decades against experimental measurements for an extensive variety of target elements and compounds, a wide selection of incident ions, and a large range of ion energies, extending from $\mathrm{eV}$ to hundreds of $\mathrm{MeV}$. SRIM calculations relatively rapidly led to the conclusion that low- $Z$ materials are preferable for the conducting layers. This is because they exhibit the lowest ratio of straggling to total range of any materials. This, in turn, provides for maximum energy resolution of the detector as the lower straggling diminishes the spread in the final deposition depth distribution of the incident ions. Aluminum was chosen as the conductive material because it is the lowest- $Z$ conducting material that did not present any significant health hazards or chemical reactivity concerns in the detector fabrication or operation environments. The choice of aluminum for the conducting layers allowed for the 
isolation of deuteron energies differing by only $15 \mathrm{keV}$, in this case the one-third and one-half neutral beam ions in NSTX-U. Silicon dioxide was chosen as the insulator because of the ease with which it can be selectively etched when used in conjunction with aluminum. The insulating layers were designed to be as thin as possible $(100 \mathrm{~nm})$ without significant risk of shorts between adjacent foils for the NTSX-U design and to be twice as thick $(200 \mathrm{~nm})$ for the JET design. This thicker choice for JET was made to further reduce the likelihood of insulating layer failure, since the $\mathrm{MeV}$ ions to be detected there are substantially more penetrating than the $30-90 \mathrm{keV}$ deuterons in NSTX-U and a greater total detector thickness can be tolerated. In both cases, the thicknesses of the layers were chosen by varying those in the SRIM model until the different ion energy components were found to stop in separate conducting layers.

\section{NSTX-U design}

The NSTX-U design consists of four aluminum conductors alternating with insulators. The thicknesses of the aluminum portions are $100 \mathrm{~nm}, 200 \mathrm{~nm}, 200 \mathrm{~nm}$, and $400 \mathrm{~nm}$, in the order encountered by an incident ion. An additional aluminum layer would be included at the back of the detector to measure intrinsic noise of the system so it can be subtracted from the data collected by the other conductive layers. The insulating layers are all $100 \mathrm{~nm}$ thick. The first aluminum coating is designed to screen the rest of the detector from UV light emitted by the plasma, which could cause false readings by inducing photoelectric electron loss. The remaining aluminum layers are for the detection of $30 \mathrm{keV}, 45 \mathrm{keV}$ and $90 \mathrm{keV}$ deuterons, respectively.

[---Place Fig. 1 here---]

Fig. 1 shows the deposition vs. depth of $90 \mathrm{keV}$ full energy beam deuterium beam ions in the detector. Nearly all the deposition is in the third conductive layer, as desired. Note that for the NSTX-U detector, the insulators are modeled as a composite of two $30 \mathrm{~nm}$ silicon dioxide layers sandwiching a $40 \mathrm{~nm}$ silicon nitride layer. This construction was chosen for modeling based upon a suggestion that deposition of the dissimilar materials would prevent propagation of crystal defects during the insulating layer deposition, hence providing a higher likelihood of electrically isolated layers. For this reason, the figures here show the insulators subdivided into three layers each. Alternatively, if silicon dioxide layers could be deposited without defect and the silicon nitride layers were not needed, then a single layer $110 \mathrm{~nm}$ thick could replace those shown here and would provide the same stopping power. Fig. 2 displays the deposition of the 45 $\mathrm{keV}$ half energy deuterons in the same NSTX-U structure shown in Fig. 1. Similarly, Fig. 3 depicts the deposition of the $30 \mathrm{keV}$ one-third energy ions in the structure, showing that the majority of these ions stop in the third layer. Due to the small variation between the range of the 
177 [Place Fig. 6 here---]

[Place Fig. 2 here---]

\section{[Place Fig. 3 here---]}

[Place Fig. 4 here---]

5 JET design

[Place Fig. 5 here---] the designated layer.

$30 \mathrm{keV}$ and $45 \mathrm{keV}$ ions, and the relatively large straggling, a fair portion (about 41\%) of the 30 $\mathrm{keV}$ deuterons will stop in the second insulating layer instead of the designated foil. It is hypothesized that this will cause the forward side of the insulator to become positively biased, which will in turn pull electrons out of the $30 \mathrm{keV}$ foil, leading to the same current that would have occurred if the ions had stopped in the foil. If this hypothesis is incorrect, the performance of the detector could suffer considerably. The ions stopping in the silicon dioxide will, over time, lead to significant damage in the insulator, including cracking and void formation, which could cause shorts between the foils. ${ }^{14,15}$ This will limit the effective lifespan of the detectors.

Fig. 4 depicts the fraction of each energy component of the incident beam that appears in each detector layer, including the intervening insulators. As can be seen in this figure, the design does a fairly good job of causing the different energy components of the beam to be deposited into different aluminum layers in the device. The principal exception to this is the second insulator, which captures $\sim 30 \%$ each of the 30 and $45 \mathrm{keV}$ beam ions.

The JET design consists of eight aluminum layers alternated with silicon dioxide, with a protective gold front coating. The thicknesses of the aluminum layers are $600 \mathrm{~nm}, 1100 \mathrm{~nm}$, $1200 \mathrm{~nm}, 1400 \mathrm{~nm}, 1600 \mathrm{~nm}, 1900 \mathrm{~nm}, 2100 \mathrm{~nm}$, and $2300 \mathrm{~nm}$, in the order encountered by an incident ion. An additional conductive layer will be included at the back of the detector to measure intrinsic noise of the system so it can be subtracted from the data collected by the other layers. The insulating layers are all $200 \mathrm{~nm}$ thick and the gold coating is $100 \mathrm{~nm}$ thick. The layer thicknesses were designed so as to have a bin size of $0.5 \mathrm{MeV}$, e.g. the first conductive layer would collect ions from $0.25 \mathrm{MeV}$ to $0.75 \mathrm{MeV}$. An example of the performance of this is shown in Fig. 5, where all the current from a population of $0.5 \mathrm{MeV}$ alphas stops in this layer.

The modeled performance of the detector when subjected to $3.0 \mathrm{MeV}$ alpha particles is shown in Fig. 6. As in the previous case, the entire population of these particles stops completely within 
179 Finally, Fig. 7 displays the computed deposition profile of $3.5 \mathrm{MeV}$ alphas in the detector, 180 where, again, all stop in their designed layer.

181 [Place Fig. 7 here---]

182 The SRIM simulations for the other alpha energies, in steps of $0.5 \mathrm{MeV}$, yield results very 183 similar to those depicted in Figs. 6 and 7, but these plots are omitted here for the sake of brevity. 184 The insulator thickness for the JET detector was increased relative to the NSTX-U design, to 185 decrease the probability of pinhole formation that could lead to shorts between conductors. 186 Because this detector is geared toward the collection of much more penetrating $\mathrm{MeV}$ ions, this 187 increase of thickness would have little effect on the overall resolution for this design. Due to the 188 sheer number of layers in the design, even a low failure rate per layer would cause a 189 prohibitively large failure rate for the detectors as a whole. The same procedure used to optimize 190 It should be noted that all the SRIM calculations have been done assuming the ions to be normally incident upon the detector surface. In practice, incidence at angles even $30^{\circ}$ off normal causes only a very modest broadening of the deposition profiles. However, this does point to the need for entrance apertures for these detectors to limit the angle of incidence of the entering ions. The JET detector already incorporates an aperture system to accomplish this.

Deposition thin layers of the materials and layer thicknesses mentioned herein can be readily accomplished by sputtering or evaporation and is commonly done in the production of semiconductor devices and in the production of optical coatings, hence should present no undue problems for use in the detectors described here.

The detectors described here, whether comprised of discrete foils or deposited layers, must operate near the plasma, an environment that is very electrically noisy. Ref. 6 displays some data from JET in this regard, showing noise levels in the nA to tens of nA range. A priori, there 204 205 In principle, the same methodology used here could be applied to the design of fast ion loss 208 detectors for virtually any magnetically confined plasma. However, it can also be concluded 209 from the NSTX-U design details given earlier, especially the information in Fig. 4, that as one 210 goes to ion energies of $\sim 50 \mathrm{keV}$ or below, it becomes more and more difficult to resolve the 211 energy distribution into anything more than two energy bins. Conceivably this limitation could 212 be overcome by constructing an array of detectors in which the layer thicknesses are varied 
213 fractionally from the base design, but the whole array is subjected to the same lost ion flux. In

214 this way, it might be possible to infer the incident energy resolution more precisely than is

215 possible with just a few conducting layers. The detector mounting structure and the fluxes in

216 JET would not allow for such an array of detectors. Potentially this concept could be attempted

217 in NSTX-U at some point in the future.

218 The digital data for this paper can be found at:

219 http://arks.princeton.edu/ark:/88435/dsp011v53k0334.

Acknowledgements

Discussions with G. Watson and J. Palmer of Princeton University's Micro and Nano Fabrication Laboratory are greatly appreciated. This work supported by US DoE contract DE-ACO2$09 \mathrm{CH} 11466$.

Figure captions

Figure 1: The SRIM computed deposition profile of $90 \mathrm{keV}$ deuterons in the NSTX-U detector, shown along with the various layers of the sensor. The white bands are the aluminum layers, while the gray regions denote the insulating layers. The latter are composite structures, as described in the text, and the red lines denote the boundaries between the different layers.

Figure 2: The SRIM computed deposition profile of $45 \mathrm{keV}$ deuterons in the NSTX-U detector, shown along with the various layers of the sensor. The detector is the same one depicted in Fig. 1 and the shading and color scheme is also the same. Note that the majority of the incident ions are deposited in the third conductive layer (second active layer), as desired.

Figure 3: The SRIM computed deposition profile of $30 \mathrm{keV}$ deuterons in the NSTX-U detector, shown along with the various layers of the sensor. The detector is the same one depicted in Fig. 1 and the shading and color scheme is also the same. Note that the majority of the incident ions are deposited in the second conductive layer (first active layer), as desired. 
Figure 4: The SRIM computed deposition fractions of the 30, 45, and $90 \mathrm{keV}$ deuterons in the various layers of the NSTX-U detector, shown along with the various layers of the sensor. $30 \mathrm{keV}$ ions stop predominantly in foil 2, $45 \mathrm{keV}$ ions in foil 3 and $90 \mathrm{keV}$ ions in foil 4, as desired.

Figure 5: The SRIM computed deposition profile of $0.5 \mathrm{MeV}$ alpha particles in the JET detector, shown along with the various layers of the sensor. As described in the text, the detector is comprised of aluminum conducting layers and silicon dioxide insulating layers. The white regions represent the conductive aluminum layers, with the exception of the leftmost one, which is a gold surface coating. The shading and red lines are the insulating layers. All of the $0.5 \mathrm{MeV}$ alphas stop in the first active layer.

Figure 6: The SRIM computed deposition profile of 3.0 MeV alpha particles in the JET detector, shown along with the various layers of the sensor. The coloring and shading are as in the previous figures. All the alphas of this energy stop in the sixth active foil.

Figure 7 The SRIM computed deposition profile of $3.5 \mathrm{MeV}$ alpha particles in the JET detector, shown along with the various layers of the sensor. The coloring and shading are as in the previous figures. All the alphas of this energy stop in the seventh active foil.

\section{References}

${ }^{1}$ M. Tuszewski and J. P. Roubin, Nucl.Fusion 28, 499 (1988).

${ }^{2}$ O.N. Jarvis, et al., Fus. Tech. 39, 84 (2001).

${ }^{3}$ D. S. Darrow, et al., Rev. Sci. Instrum. 75, 3566 (2004).

${ }^{4}$ V. Basiuk et al, Nucl. Fusion 44, 181 (2004).

${ }^{5}$ Y. B. Zhu, W. W. Heidbrink and L. D. Pickering, Nucl. Fusion 50, 084024 (2010).

${ }^{6}$ D. S. Darrow, et al., Rev. Sci. Instrum. 81, 10D330 (2010)

${ }^{7}$ S. Baeumel, A. Werner, R. Semler, and S. Mukherjee, et al., Rev. Sci. Instrum. 75, 3563 (2004).

${ }^{8}$ F.E. Cecil, et al., Rev. Sci. Instrum. 70, 1149 (1999).

${ }^{9}$ F.E. Cecil, et al, Rev. Sci. Instrum. 72, 792 (2001).

${ }^{10}$ R. Aymar, P. Barabaschi, and Y. Shimomura, Plasma Phys. Control. Fusion 44, 519 (2002). 
$275{ }^{11}$ J. E. Menard, et al., Nucl. Fusion 52, 083015 (2012).

$276 \quad{ }^{12}$ F.E. Cecil, et al, Rev. Sci. Instrum. 74, 1747 (2003).

$277{ }^{13}$ The code SRIM 2008, Stopping and Range of Ions in Matter, was written by J. F. Zeigler and J. 278 Biersack, IBM-Research, Yorktown Heights, New York 10598. (SRIM is freely available at 279 http://www.srim.org.)

$280{ }^{14}$ J. Kim, et al., J. Korean Physical Soc. 43, 582, (2003).

$281 \quad{ }^{15}$ W. Primack, J. Nucl. Mat. 53, 238 (1974). 


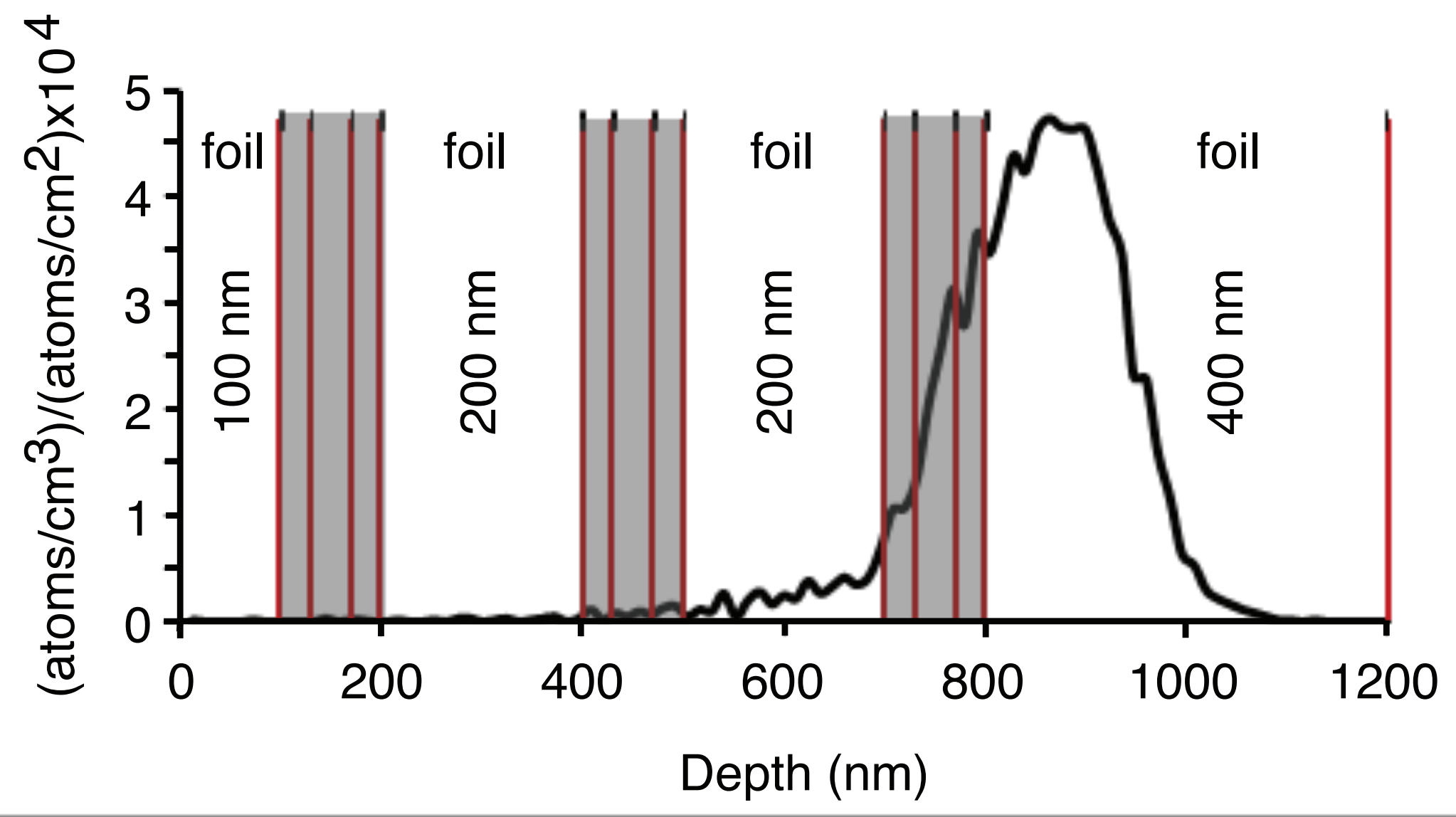




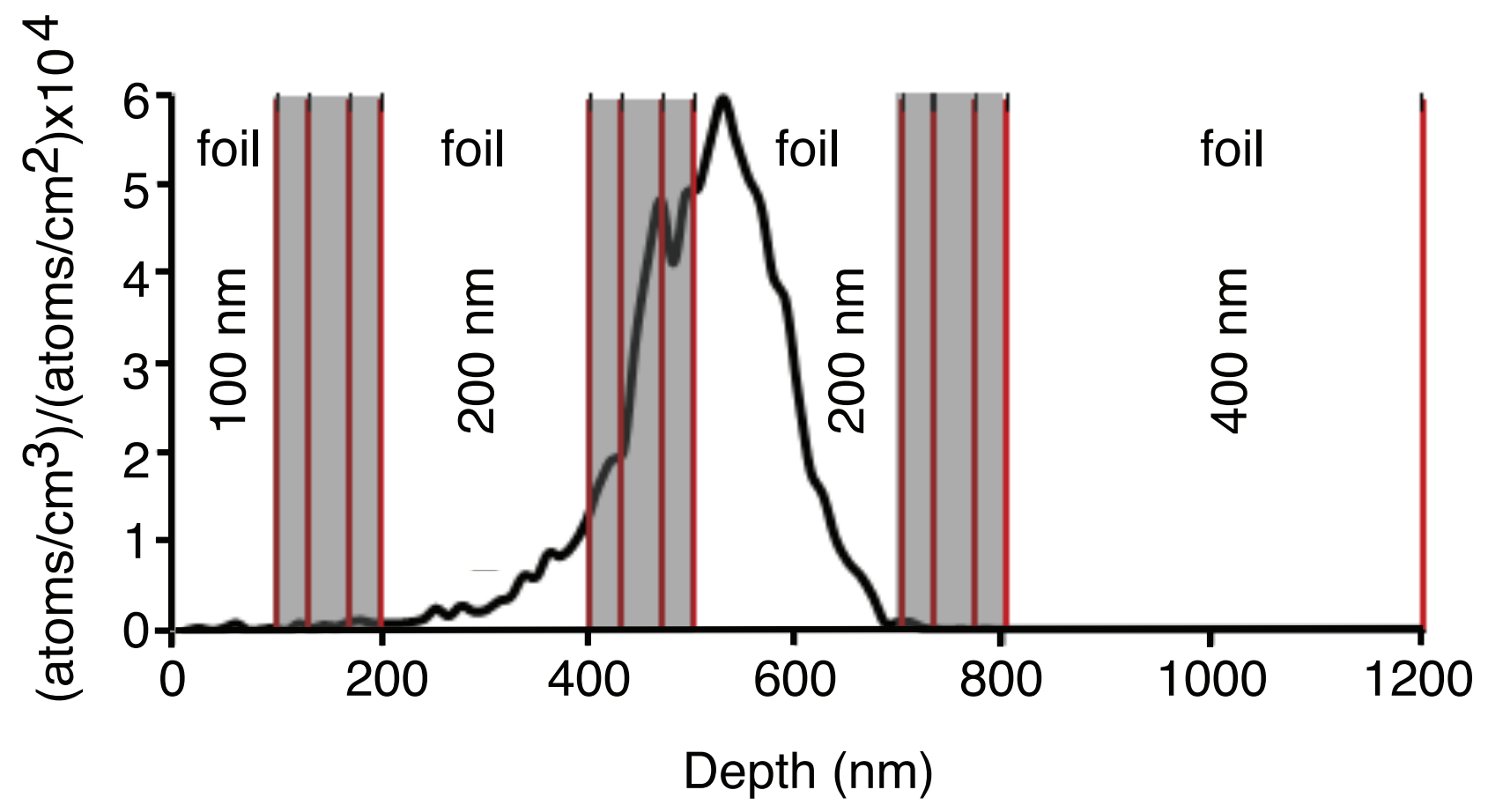




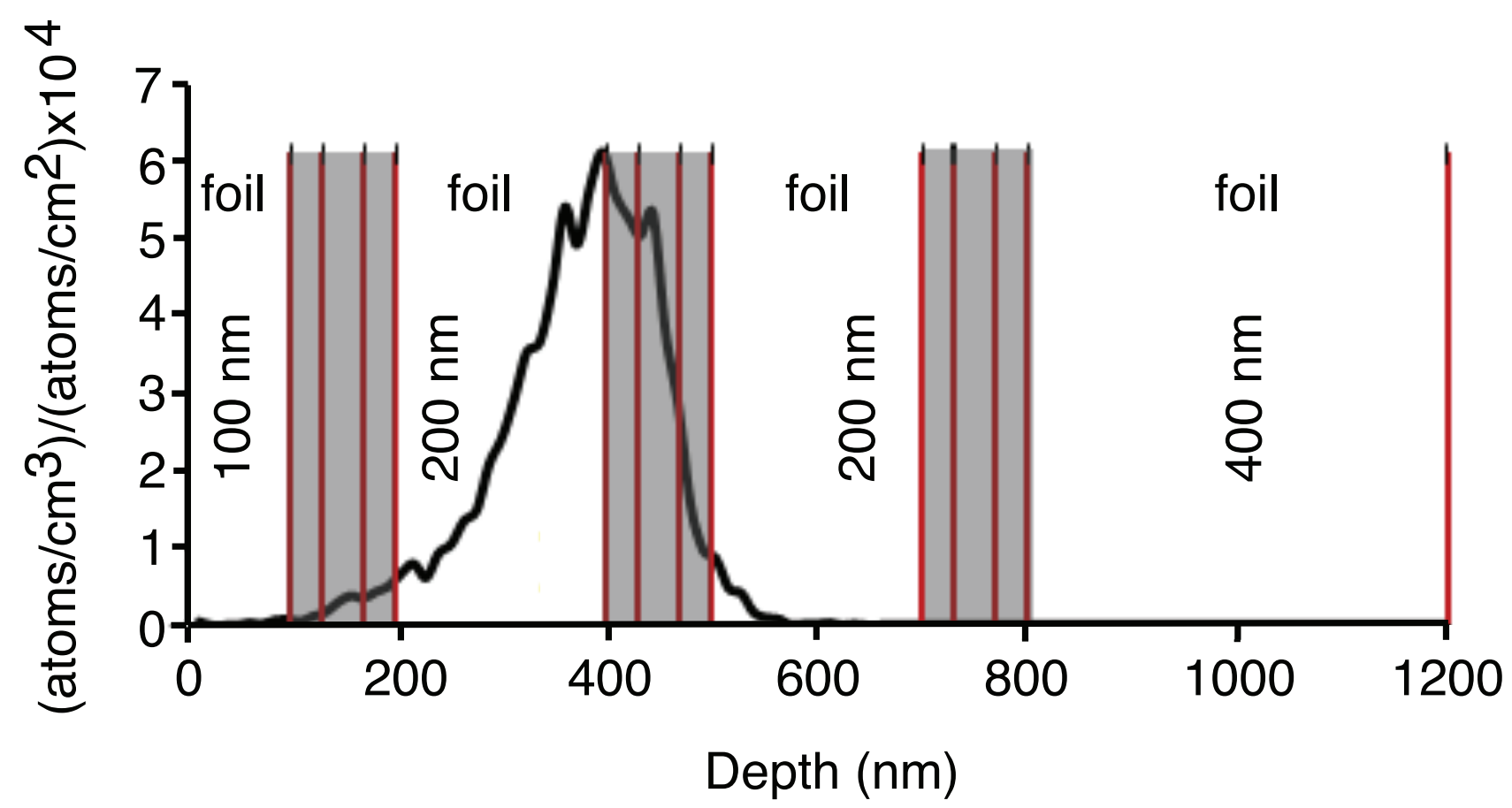




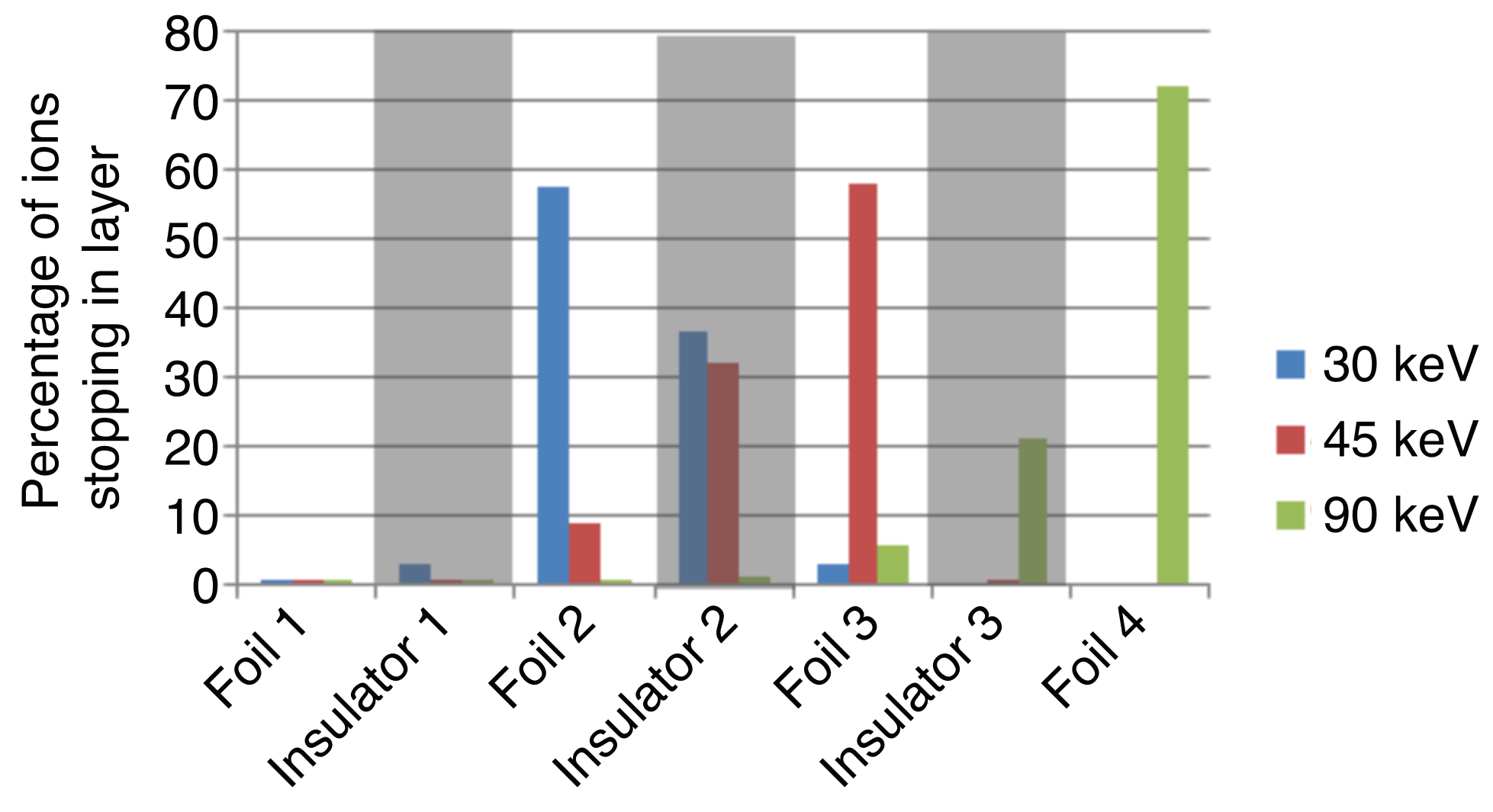




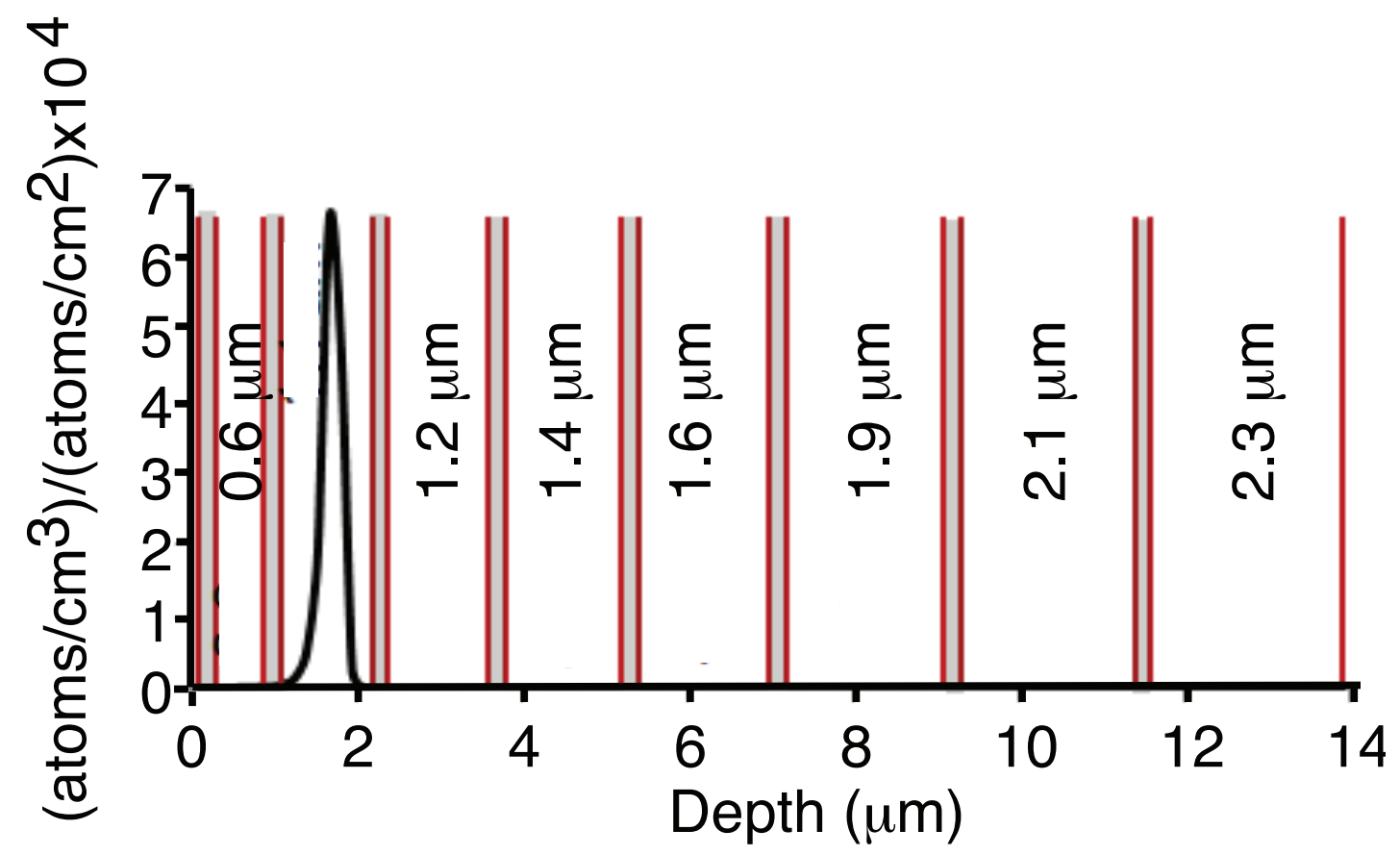




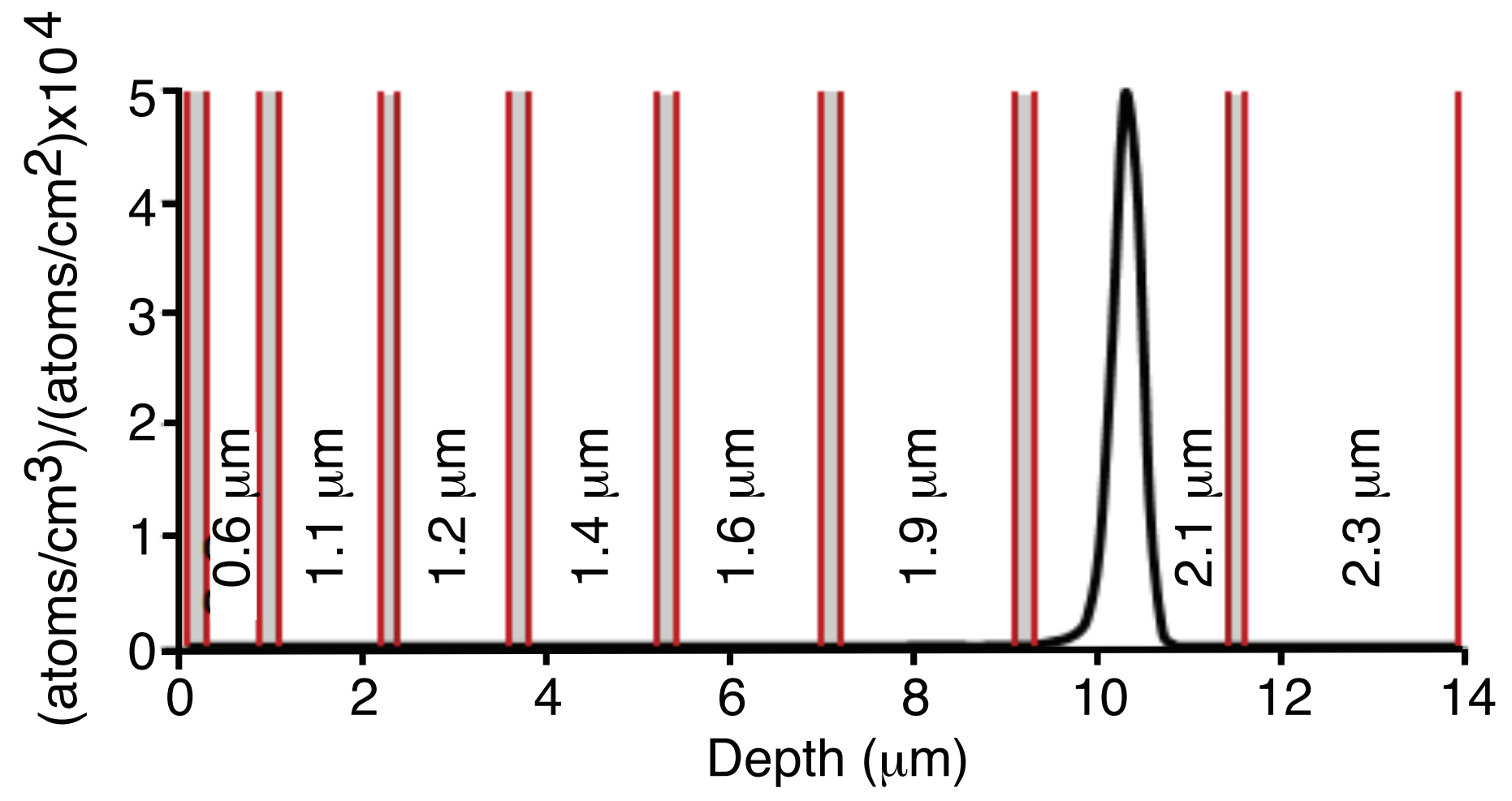




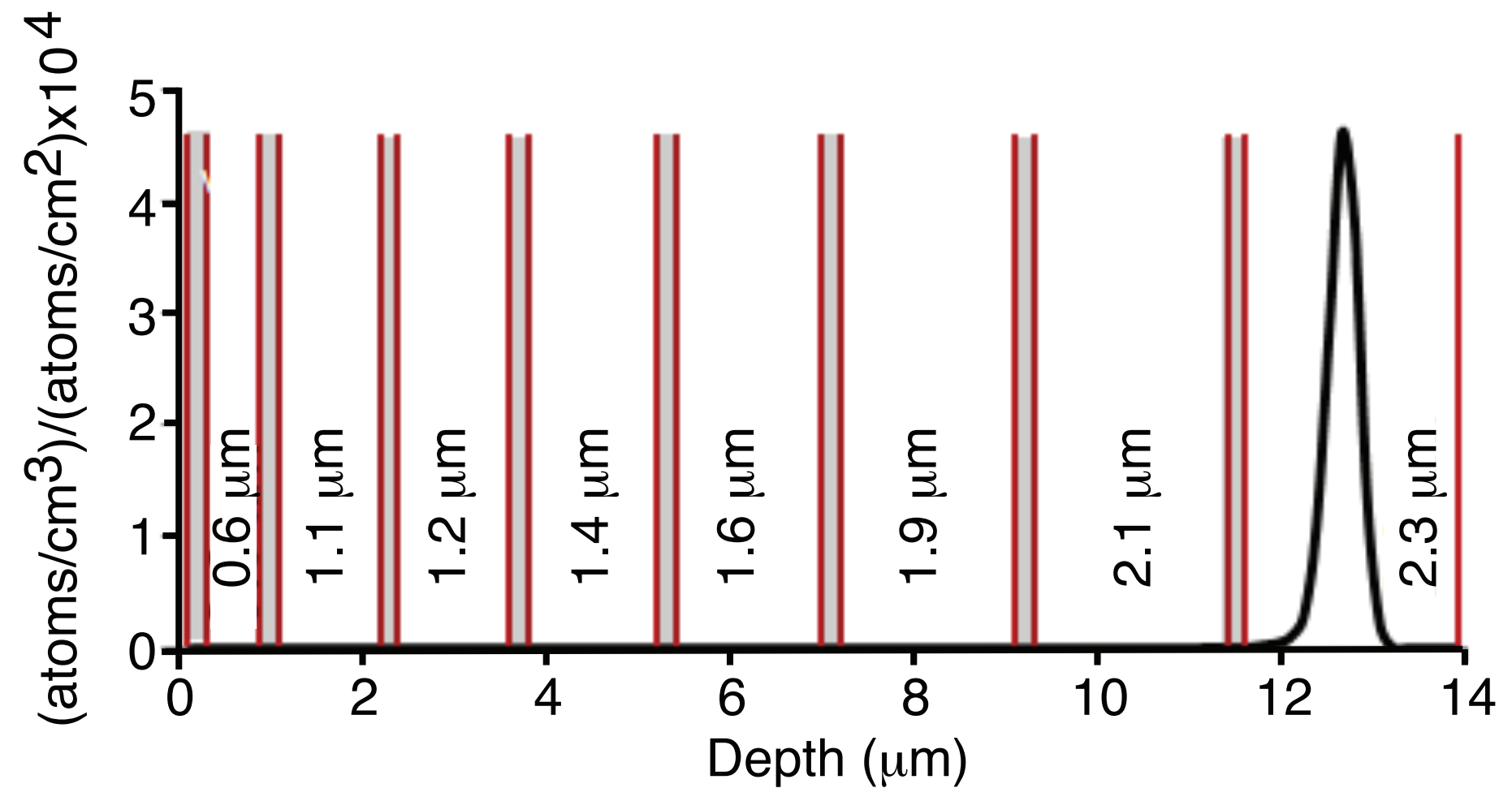

